\title{
Verpflichtet das Völkerrecht zur Einführung von Kinderrechten ins Grundgesetz?
}

\section{- Ein Statement in 18 Thesen -}

\author{
Stefanie Schmahl
}

\begin{abstract}
s
Die Einführung von Kinderrechten ins Grundgesetz ist Gegenstand intensiver Diskussionen. In der Debatte wird immer wieder vorgebracht, die Einführung von Kinderrechten ins Grundgesetz sei völkerrechtlich geboten. Doch trifft diese Behauptung tatsächlich zu? Und entspricht die geplante Ergänzung von Art. 6 GG den Erwartungen, die sich aus dem UN-Übereinkommen über die Rechte des Kindes ergeben?

The introduction of children's rights into the Basic Law is the subject of intensive discussions. In the debate, it is repeatedly argued that the introduction of children's rights into the Basic Law is required by international law. But does this assertion actually apply? And does the planned amendment of Article 6 of the Basic Law correspond to the expectations resulting from the UN Convention on the Rights of the Child?
\end{abstract}

\section{Politischer Wille zur Einführung von Kinderrechten in das Grundgesetz}

1. In ihrem Koalitionsvertrag für die 19. Legislaturperiode haben sich CDU, CSU und SPD auf die Einführung von Kinderrechten ins Grundgesetz verständigt. ${ }^{1}$ Zur Umsetzung dieses Auftrags wurde im Jahr 2018 eine Bund-Länder-Arbeitsgruppe „Kinderrechte ins Grundgesetz“ errichtet, deren Abschlussbericht seit dem 14. Oktober 2019 vorliegt. Darin enthält sich die Arbeitsgruppe im Ergebnis zwar einer eindeutigen Empfehlung, erörtert aber die unterschiedlichen substanziellen Varianten der Einführung eines spezifischen Kindergrundrechts sowie seiner potenziellen Regelungsstandorte, etwa in Art. 6 GG oder in einem neu hinzuzufügenden Art. 2 a GG. ${ }^{2}$ Auf dieser Grundlage sieht der inzwischen fertiggestellte Referentenentwurf des Bundesministeriums der Justiz und für Verbraucherschutz (BMJV) vom 22. November 2019 folgende Ergänzung des Art. 6 GG um einen neuen Absatz 1 a vor: ${ }^{3}$ „Jedes Kind hat das Recht auf Achtung, Schutz und Förderung seiner Grundrechte einschließlich seines Rechts auf Entwicklung zu einer eigenverantwortlichen Persönlichkeit in der sozialen Gemeinschaft. Das

1 Ein neuer Aufbruch für Europa. Eine neue Dynamik für Deutschland. Ein neuer Zusammenhalt für unser Land, Koalitionsvertrag zwischen CDU, CSU und SPD, 19. Legislaturperiode, vom 12.3.2018, S. 21. www.bundesregierung.de/breg-de/themen/koalitionsvertrag-zwischen-cdu-csu-und-spd-195906.

2 Abschlussbericht der Bund-Länder-Arbeitsgruppe „Kinderrechte ins Grundgesetz“, vom 14.10.2019, S. 111 ff. www.bmjv.de/SharedDocs/Downloads/DE/News/PM/102519_Abschlussbericht_Kinderrechte.pdf ? blob $=$ publicationFile \&v $=2$.

3 Referentenentwurf des Bundesministeriums der Justiz und für Verbraucherschutz: Entwurf eines Gesetzes zur Änderung des Grundgesetzes zur ausdrücklichen Verankerung der Kinderrechte vom 22.11.2019 (noch nicht im Internet erhältlich; der Entwurf liegt der Verf. vor). 
Wohl des Kindes ist bei allem staatlichen Handeln, das es unmittelbar in seinen Rechten betrifft, angemessen zu berücksichtigen. Jedes Kind hat bei staatlichen Entscheidungen, die seine Rechte unmittelbar betreffen, einen Anspruch auf rechtliches Gehör."

2. Immer wieder wird vorgebracht, die Einführung von Kinderrechten ins Grundgesetz sei völkerrechtlich induziert, ja gar geboten. ${ }^{4}$ Doch trifft diese Behauptung tatsächlich zu? Und entspricht der vorgelegte Referentenentwurf des BMJV den allfälligen Erwartungen, die sich aus dem Übereinkommen über die Rechte des Kindes vom 20. November 1989 (UN-Kinderrechtskonvention; UN-KRK) an die Vertragsstaaten ergeben? Diese beiden Fragen stehen im Mittelpunkt der nachfolgenden Überlegungen.

\section{Zielsetzung, Regelungsgehalt und Bedeutung der UN-Kinderrechtskonvention}

3. Die UN-Kinderrechtskonvention ist für Deutschland im Jahr 1992 in Kraft getreten ${ }^{5}$ und steht, wie alle völkerrechtlichen Abkommen, bei denen die Bundesrepublik Deutschland Vertragspartei ist, nach Maßgabe des Art. 59 Abs. 2 Satz 1 GG im normhierarchischen Rang eines einfachen Bundesgesetzes. Damit kann die UN-Kinderrechtskonvention verfassungsrechtliche Vorgaben nicht verdrängen. Das Bundesverfassungsgericht hat aber klargestellt, dass das Übereinkommen bei der Auslegung der Grundrechte und des Rechtsstaatsprinzips des Grundgesetzes im Wege der völkerrechtsfreundlichen Auslegung zu berücksichtigen ist. ${ }^{6}$

4. Regelungsgehalt und Telos der UN-Kinderrechtskonvention sind unzweideutig: Das Übereinkommen zielt auf die Anerkennung von Partizipationsrechten für Kinder, d.h. für Menschen, die geboren sind, aber das 18. Lebensjahr noch nicht vollendet haben. ${ }^{7}$ Kinder sollen nicht mehr als Objekte ihrer Eltern oder anderer Sorgeberechtigter, sondern als eigenständige Akteure und Rechtssubjekte wahrgenommen werden. Dies zeigt sich in zahlreichen Vorschriften des Übereinkommens, insbesondere an der generellen Schlüsselnorm des Art. 12 UN-KRK zu den Mitspracherechten des Kindes in allen es betreffenden Angelegenheiten. ${ }^{8}$ Auch das Kindeswohl, dem gemäß Art. 3 UN-KRK vorrangige Bedeutung in allen Angelegenheiten zukommt und das darüber hinaus in verschiedenen Vorschriften des Übereinkommens spezifiziert wird, ist wesentlich von der Meinung und dem individuellen Willen des Kindes geprägt. ${ }^{9}$ Der UNKinderrechtsausschuss geht davon aus, dass dem Wohl des Kindes am besten entsprochen

4 Vgl. aus jüngerer Zeit etwa Hohmann-Dennhardt, C., Kinderrechte ins Grundgesetz - warum?, FPR 2012, S. 185; Benassi, G., Kinderrechte ins Grundgesetz - alternativlos!, ZRP 2015, S. 24; ders., Deutsche Rechtsprechung vs. UN-Kinderrechtskonvention?, DVB1. 2016, S. 617 (621); ders./Eichholz, R., Grundgesetz und Kinderrechte, DVB1. 2017, S. 614; Hofmann, R./Donath, P., Gutachten bezüglich der ausdrücklichen Aufnahme von Kinderrechten in das Grundgesetz nach Maßgabe der Grundprinzipien der UN-Kinderrechtskonvention, 2017, S. $11 \mathrm{ff} ., 41$.

5 BGBl. 1992 II S. 121; Bekanntmachung vom 10.7.1992, in: BGB1. 1992 II S. 990.

6 Vgl. BVerfG, Beschluss vom 5. Juli 2013, 2 BvR 708/12, Rn. 21.

7 Vorgeburtliche Rechte etabliert die Kinderrechtskonvention nicht. Insoweit sind die travaux préparatoires eindeutig, vgl. Alston, $P$., The Unborn Child and Abortion under the Draft Convention on the Rights of the Child, Human Rights Quarterly 12 (1990), S. 156.

8 Eingehend hierzu UN-Kinderrechtsausschuss, General Comment No. 12: The right of the child to be heard, UN-Dok. CRC/C/GC/12, 2009, Rn. $1 \mathrm{ff}$.

9 Vgl. Wyttenbach, J., Übereinkommen über die Rechte des Kindes (CRC), in: Pollmann, A./Lohmann, G. (Hrsg.), Menschenrechte. Ein interdisziplinäres Handbuch, 2012, S. 317 (317); Eichholz, R., Der Vorrang des Kindeswohls: Die Bedeutung von Art. 3 Absatz 1 der UN-Kinderrechtskonvention für die deutsche Rechtsprechung, 2015, S. 16 f. 
wird, wenn seine Meinung zu den es betreffenden Angelegenheiten gehört und in der Entscheidungsfindung berücksichtigt wird. ${ }^{10}$ Mit anderen Worten ist das Mitspracherecht des Kindes in allen es berührenden Angelegenheiten wesentlicher Ausdruck des Kindeswohlprinzips. Während Art. 3 UN-KRK darauf zielt, dass das Kindeswohl bei allen zu ergreifenden Maßnahmen hinreichend, ja vorrangig berücksichtigt wird, gibt Art. 12 UN-KRK die Methode zur Feststellung des Kindeswohls vor. ${ }^{11}$ Das Wohl des Kindes kann deshalb nur dann zutreffend ermittelt und angewandt werden, wenn die subjektive Rechtsstellung des Kindes und seine daraus folgenden Partizipationsrechte gewahrt sind.

5. Mit diesem eindeutig subjektivrechtlichen Ansatz weist die UN-Kinderrechtskonvention als internationales Antidiskriminierungsinstrument einen klaren emanzipatorischen Charakter auf, der allerdings im Verhältnis zu anderen Menschenrechtsabkommen, die verletzliche Gruppen in den Blick nehmen, abgeschwächt ist. ${ }^{12}$ Im Regelfall wächst nämlich ein Mensch aus der Lebensphase Kindheit durch Zeitablauf heraus und muss sich - anders als z.B. Frauen und Menschen mit Behinderungen - nicht grundsätzlich aus der defensiven Objektrolle befreien. ${ }^{13}$ Außerdem ist ein Kind, zumal ein Säugling oder Kleinkind, auf Hilfe und Unterstützung durch Erwachsene notwendig angewiesen, weshalb Art. 12 UN-KRK nur von einem dem Alter und Reifegrad des Kindes entsprechenden Mitspracherecht ausgeht.

6. Zudem bedeutet die Anerkennung des Kindes als Rechtssubjekt nicht, dass Gemeinwohlbelange und Rechte anderer Personen gegenüber den Interessen des Kindes automatisch zurücktreten müssten. Kinder sind nicht die einzigen Grundrechtsträger. Mit Bedacht ist das Kindeswohl in der zentralen Norm des Art. 3 Abs. 1 UN-KRK daher auch nicht als vorbehaltloses Recht gefasst, sondern wird dort nur als ein Gesichtspunkt genannt, der vorrangig zu berücksichtigen ist. Das Wohl des Kindes muss zwar stets eine herausragende Leitlinie bei der Abfassung, Auslegung und Anwendung nationaler Rechtsvorschriften darstellen. ${ }^{14}$ Eine ähnlich weitreichende Vorrangklausel mit unmittelbarer Anwendbarkeit ${ }^{15}$ kennt kein anderer Menschenrechtsvertrag. ${ }^{16}$ Dennoch kommt dem Kindeswohl keine absolute Priorität gegenüber anderen Belangen und Rechtsträgern zu. ${ }^{17}$ Ganz im Gegenteil trägt die UN-Kinderrechtskonven-

10 UN-Kinderrechtsausschuss, General Comment No. 14: The right of the child to have his or her best interests taken as a primary consideration, UN-Dok. CRC/C/GC/14, 2013, Rn. 43.

11 Vgl. UN-Kinderrechtsausschuss, General Comment No. 14 (Anm. 10), Rn. 43 f. sowie UN-Kinderrechtsausschuss, Auffassungen vom 27.9.2018, Y.B. and N.S. v. Belgium, UN-Dok. CRC/C/79/D/12/2017, Rn. 8.8.

12 Schmahl, S., Kinderrechte im internationalen Recht in Geschichte und Gegenwart, in: Richter, I./Krappmann, L./Wapler, F. (Hrsg.), Kinderrechte. Handbuch des deutschen und internationalen Kinder- und Jugendrechts, 2020, i.E.

13 Zutreffend Wapler, F., Kinderrechte und Kindeswohl, 2015, S. 401.

14 UN-Kinderrechtsausschuss, General Comment No. 14 (Anm. 10), Rn. 37. Vgl. auch Lorz, R. A., Der Vorrang des Kindeswohls nach Art. 3 der UN-Kinderrechtskonvention in der deutschen Rechtsordnung, 2003, S. $24 \mathrm{ff}$.

15 Dazu Schmahl, S., Kinderrechtskonvention mit Zusatzprotokollen, Handkommentar, 2. Aufl. 2017, Art. 3 Rn. 5 und Rn. 16. Auch die jüngere Fachjudikatur versteht Art. 3 Abs. 1 KRK vermehrt als unmittelbar anwendbares Recht, vgl. VG Saarland, Urteil vom 4.11.2016, 3 K 921/15, Rn. 39; VG Berlin, FamRZ 2018, S. 399 (401).

16 Vgl. Dederer, H.-G., Kinderrechte auf internationaler und supranationaler Ebene. Bestandsaufnahme und Ausblick, in: Uhle, A. (Hrsg.), Kinder im Recht. Kinderrechte im Spiegel der Kindesentwicklung, 2019, S. 287 (304).

17 Schmahl (Anm. 15), Art. 3 Rn. 7; vgl. auch Freeman, M., Article 3, in: Alen, A. et al. (Hrsg.), A Commentary on the United Nations Convention on the Rights of the Child, Band 3, 2007, S. 5, 60 f.; Eekelaar, J./ Tobin, J., Article 3, in: Tobin, J. (Hrsg.), The UN Convention on the Rights of the Child, 2019, S. 73 (96). 
tion auch den Rechten und Pflichten der Eltern und sonstiger Sorgeberechtigten Rechnung. Das allgemeine Erziehungsrecht der Eltern kommt etwa in Art. 3 Abs. 2, Art. 5, Art. 14 Abs. 2 und in Art. 18 Abs. 1 UN-KRK zum Ausdruck. In verschiedenen Bereichen gesteht damit die Konvention den Eltern ein Erziehungsprimat zu, das freilich seinerseits im Sinne einer dienenden Elternverantwortung auf die Berücksichtigung des Kindeswohls unter Einbeziehung des Kindeswillens verpflichtet ist. ${ }^{18}$ Auch der UN-Kinderrechtsausschuss stellt ausdrücklich klar, dass der Terminus ,vorrangig“ in Art. 3 Abs. 1 UN-KRK nicht mit „Vorrang der Interessen des Kindes vor allen anderen Belangen" gleichzusetzen sei. ${ }^{19}$

7. Damit greift die Konvention das Spannungsfeld zwischen den Belangen des Kindes, den Rechten seiner Eltern und anderen (auch öffentlichen) Interessen auf, das in der Lebensphase Kindheit häufig vorfindlich ist. Die Meinung des Kindes ist in allen es betreffenden Angelegenheiten konsultativ zu berücksichtigen, ohne ihm zugleich die soziale Eingebundenheit in das Elternhaus und die Gesellschaft sowie den notwendigen Schutz vor Gefahren zu verwehren. Aus Art. 3 i.V.m. Art. 12 UN-KRK folgt, dass Kinder von Anfang an in die sie betreffenden Entscheidungsfindungen ernsthaft und respektvoll einzubeziehen sind, auch wenn die Entscheidung letztlich von den Sorgeberechtigten getroffen wird. ${ }^{20}$ Dabei kommt dem Alter, dem Reifegrad und den zunehmenden Fähigkeiten des Kindes zentrale Bedeutung zu. ${ }^{21}$ Dem Willen des Heranwachsenden ist bei der Bestimmung des Kindeswohls größeres Gewicht einzuräumen als dem Willen eines Klein- oder Kleinstkindes. Wegen der wachsenden Selbstbestimmungsfähigkeit muss allerdings ausnahmslos jedem Kind ein alters- und reifegerechter Mindestbestand an Wahlmöglichkeiten für die Zukunft eröffnet bleiben, damit es sich unter Umständen von seinen Herkunftsbedingungen lösen kann. ${ }^{22}$

8. Trotz oder vielleicht gerade wegen dieser innovativen kinderrechtlichen Perspektive verfügt die UN-Kinderrechtskonvention nur über schwache Durchsetzungsmechanismen. Zwar verlangt Art. 4 Satz 1 UN-KRK, dass die Konvention im innerstaatlichen Rechtsraum praktische Wirksamkeit erlangt. ${ }^{23}$ Auch bestehen keine Zweifel an der Justiziabilität des Übereinkommens und der unmittelbaren Anwendbarkeit vieler - nicht aller - in ihm niedergelegter Garantien, insbesondere, soweit diese klassische bürgerliche Rechte garantieren. ${ }^{24}$ Doch sind die Vertragsstaaten nach Art. 44 UN-KRK lediglich verpflichtet, dem UN-Kinderrechtsausschuss, einem unabhängigen Expertengremium, über die interne Verwirklichung ihrer übernommenen Vertragspflichten in regelmäßigen Abständen Bericht zu erstatten und dem Ausschuss für ein dialogisches Verfahren zur Verfügung zu stehen. Anerkennung und Anwendung des quasi-gerichtlichen Mitteilungsverfahrens, das mit dem im Jahre 2014 in Kraft getretenen 3. Fakultativprotokoll zur UN-Kinderrechtskonvention eingeführt worden ist, stehen sogar im freien Ermessen der Staaten. Eine Ratifikationspflicht besteht nicht. Die Bundesrepublik Deutschland hat

18 Hierüber besteht Einigkeit, vgl. nur Schmahl (Anm. 15), Art. 5 Rn. 5; Benassi/Eichholz (Anm. 4), S. 618; Khazova, O. A., International Children's Rights Law: Child and the Family, in: Kilkelly, U./Liefaard, T. (Hrsg.), International Human Rights of Children, 2019, S. 161 (167), jeweils m.w.N.

19 UN-Kinderrechtsausschuss, General Comment No. 14 (Fn. 10), Rn. 39.

20 Zur Unterscheidung zwischen konsultativer und dezisiver Wirkung des Kindeswillens vgl. Wapler (Anm. 13), S. $435 \mathrm{ff}$.

21 UN-Kinderrechtsausschuss: General Comment No. 14 (Anm. 10), Rn. 44.

22 Vgl. Wapler, F., Religiöse Kindererziehung. Grenzen des Rechts, RdJB 2015, S. 420 (424 und 447).

23 Dazu näher UN-Kinderrechtsausschuss, General Comment No. 5: General measures of implementation of the Convention on the Rights of the Child, UN-Dok. CRC/GC/2003/5, 2003, Rn. 22.

24 Schmahl (Anm. 15), Einleitung, Rn. 26 f.; Dederer (Anm. 16), S. 310. Ohne nähere Begründung skeptisch zur unmittelbaren Anwendbarkeit: OVG Nds., Beschluss vom 2.10.2012, 8 LS 209/11, Rn. 20 ff. 
das 3. Fakultativprotokoll freilich als einer der ersten Vertragsstaaten ratifiziert ${ }^{25}$ und damit die Möglichkeit einer Individualbeschwerde gegen deutsche Hoheitsakte vor dem UN-Kinderrechtsausschuss eröffnet.

9. Der UN-Kinderrechtsausschuss entscheidet jedoch sowohl im Berichts- als auch im Individualbeschwerdeverfahren lediglich im Wege von unverbindlichen Stellungnahmen, allgemeinen Bemerkungen und Empfehlungen. ${ }^{26}$ Eine völkerrechtliche Verantwortlichkeit der Bundesrepublik Deutschland für Defizite bei der Umsetzung dieser Stellungnahmen kommt daher nicht in Betracht, sofern diese über den konsentierten Konventionstext hinausgehen. ${ }^{27}$ Dem steht nicht entgegen, dass den empfehlenden Auffassungen des Ausschusses wegen des unabhängigen Sachverstands seiner Mitglieder hohe Autorität zukommt, der sich die Staaten schon aus Gründen des allgemeinen Rechtsgrundsatzes von Treu und Glauben nur unter besonderem Argumentationsaufwand entziehen können. ${ }^{28}$

10. Das wesentliche Verdienst der UN-Kinderrechtskonvention besteht darin, die subjektive Rechtsstellung von Kindern als eigenständige Rechteinhaber in das Bewusstsein der internationalen Staatengemeinschaft und der Gesellschaften gebracht zu haben. So lässt sich mittlerweile konstatieren, dass die spezifisch kinderrechtliche und subjektorientierte Perspektive, die der Konvention zugrunde liegt, verschiedene nachfolgende universelle wie regionale Menschenrechtsabkommen inspiriert hat und in der Judikatur internationaler Gerichte zunehmend aufgegriffen wird. ${ }^{29}$ Insbesondere für den europäischen Kontext ist von Bedeutung, dass der EGMR als für die Auslegung der EMRK verbindliches Gericht in seiner Judikatur inzwischen immer wieder auf die UN-Kinderrechtskonvention sowohl in strafrechtlichen als auch umgangsrechtlichen sowie nicht zuletzt in asylrechtlichen Streitigkeiten Bezug nimmt ${ }^{30}$ Darüber hinaus enthält die Grundrechtecharta der Europäischen Union mit Art. 24 eine spezielle Norm, die Rechte des Kindes statuiert. Dabei ist augenfällig, dass sich alle drei Absätze von Art. 24 GRCh an den Bestimmungen der UNKinderrechtskonvention, vor allem an deren Art. 3 und Art. 12 UN-KRK, als Rechtserkenntnisquelle wesentlich orientieren. ${ }^{31}$ Daher erstaunt es nicht, dass der EuGH zu Art. 24 Abs. 2 GRCh klargestellt hat, dass der dort normierte Vorrang des Kindeswohls - ebenso wie bei Art. 3 UNKRK - nicht im Sinne eines absoluten Vorrangs zu verstehen ist. ${ }^{32}$

25 BGBl. 2012 II S. 1546.

26 Vgl. Art. 45 lit. d UN-KRK und Art. 10 Abs. 5 des 3. Fakultativprotokolls zur UN-KRK. Ferner siehe $P a-$ yandeh, M., Die Individualbeschwerde zum Kinderrechtsausschuss der Vereinten Nationen. Rechtsgutachten, April 2014, S. $53 \mathrm{ff}$.

27 Dederer (Anm. 16), S. 306.

28 Näher Schmahl, S., Auswirkungen der UN-Kinderrechtskonvention auf die deutsche Rechtsordnung, RdJB 2014, S. 125 (128 f.); vgl. auch Klein, E., Allgemeine Bemerkungen der UN-Menschenrechtsausschüsse, in: Merten, D./Papier, H.-J. (Hrsg.), Handbuch der Grundrechte, Band VI/2, 2009, § 127 Rn. 27 und 31 .

29 Sogar die internationale Strafgerichtsbarkeit nimmt auf die UN-Kinderrechtskonvention Bezug; deutlich etwa Spezialgerichtshof für Sierra Leone, Appeals Chamber, Prosecutor v. Tamba Brima u.a., Urteil vom 22.2.2008, SCSL-2004-16-A, sowie Internationaler Strafgerichtshof (Trial Chamber), Prosecutor v. Thomas Lubanga Dyilo, Urteil vom 14.3.2012, ICC-01/04-01/06, Rn. $600 \mathrm{ff}$.

30 Vgl. z. B. EGMR, Sahin v. Germany, Urteil vom 8.7.2003, App. No. 30943/96, Rn. 64; Neulinger and Shuruk v. Switzerland, Urteil vom 6.7.2010, App. No. 41615/07, Rn. 132; Nunez v. Norway, Urteil vom 28.6.2011, App. No. 55597/09, Rn. 84.

31 Vgl. nur Hölscheidt, S., in: Meyer, J. (Hrsg.), Charta der Grundrechte der EU. Kommentar, 5. Aufl. 2019, Art. 24 Rn. 2 ff.; Schmahl, S., Gleichheitsgarantien, in: Grabenwarter, C. (Hrsg.), Enzyklopädie Europarecht, Band 2, 2. Aufl. 2020 (i.E.), § 21 Rn. 104.

32 EuGH, MA, BT, DA v. Secretary of State for the Home Department, Urteil vom 6.6.2013, Rs. C-648/11, ECLI:EU:C:2013:367, Rn. 57 ff. Vgl. auch EuGH, Maahanmuuttovirasto, Urteil vom 6.12.2012, 
11. Des Weiteren hat die UN-Kinderrechtskonvention die Fortentwicklung nationaler Rechtsund Verfassungsordnungen beeinflusst. So haben manche Staaten, darunter Spanien, Finnland, Polen und Österreich, inzwischen Bestimmungen in ihre Verfassungen aufgenommen, die Kinderrechte betonen und stärken. ${ }^{33}$ Der Vertragstext der UN-Kinderrechtskonvention selbst fordert zwar keine verfassungsrechtliche Implementierung ihrer wesentlichen Regelungen. ${ }^{34}$ Dennoch betont der UN-Kinderrechtsausschuss, dass er eine Aufnahme der zentralen Prinzipien der Konvention in die nationalen Verfassungen begrüßen würde. ${ }^{35}$ Dies hat er mehrfach auch im Blick auf die deutsche Rechtsordnung unterstrichen. Dabei hat er sogar angemahnt, dass der UN-Kinderrechtskonvention Vorrang gegenüber einfachem Bundesrecht zuerkannt werden solle. ${ }^{36}$

\section{Keine völkerrechtliche Notwendigkeit zur Einführung von Kinderrechten in die Verfassung}

12. Dieses (rechtspolitische) Drängen des UN-Kinderrechtsausschusses auf eine Verankerung von Kinderrechten im Grundgesetz oder auf einen sonstigen rechtlichen Mechanismus, der den Kinderrechten Vorrang vor einfachem Recht verschafft, hat keine Grundlage in der Konvention oder in einer anderweitig bestehenden völkerrechtlichen Pflicht. ${ }^{37}$ Soweit sich die Befürworter der Einführung einer Kinderrechtsklausel in die Verfassung auf die Implementierungspflicht des Art. 4 UN-KRK berufen, ${ }^{38}$ unterliegen sie einem Missverständnis. Dem Wortlaut von Art. 4 Satz 1 UN-KRK, wonach ,[d]ie Vertragsstaaten [...] alle geeigneten Gesetzgebungs-, Verwaltungs- und sonstigen Maßnahmen zur Verwirklichung der in diesem Übereinkommen anerkannten Rechte [treffen]“", lässt sich nur entnehmen, dass ein Implementierungsauftrag für die Vertragsstaaten besteht, nicht aber, auf welche Weise dieser zu erfüllen ist. Die Implementierungs- und Umsetzungspflicht, die sich nach Art. 4 Satz 2 UN-KRK auch auf die in der Konvention verankerten wirtschaftlichen, sozialen und kulturellen Rechte bezieht, ist auch in anderen Menschenrechtsverträgen enthalten. Sie stellt lediglich klar, dass die Vertragsstaaten den Gesamtzustand des nationalen Rechts auf das Übereinkommen einzustellen haben. Bei der Wahl der Form und Mittel gesteht Art. 4 UN-KRK den Vertragsstaaten aber Spielräume zu. ${ }^{39}$ Zwar müssen die Vertragsstaaten nach Auffassung des UN-Kinderrechtsausschusses nachweisen, dass sie ihre verfügbaren finanziellen und rechtlichen Ressourcen bestmöglich im Sinne der UN-Kinderrechtskonvention ausschöpfen. ${ }^{40}$ Auf diese Weise werden sowohl der Beurtei-

Rs. C-356/11 und C-357/11, ECLI:EU:C:2012:776, Rn. 79 f.; Zarraga v. Pelz, Urteil vom 22.12.2010, Rs. C-491/10 PPU, ECLI:EU:C:2010:828, Rn. 62 ff.

33 Vgl. Schmahl (Anm.. 15), Einleitung, Rn. 22.

34 So auch Rossa, E., Kinderrechte. Das Übereinkommen über die Rechte des Kindes im internationalen und nationalen Kontext, 2013, S. 102.

35 UN-Kinderrechtsausschuss: General Comment No. 5 (Anm. 23), Rn. 20.

36 Vgl. UN-Kinderrechtsausschuss: Concluding Observations: Germany, UN-Dok. CRC/C/15/Add.43, 1995, Rn. 21; UN-Dok. CRC/C/15/Add.226, 2004, Rn. 9 f.; UN-Dok. CRC/C/DEU/CO/3-4, 2014, Rn. 9 f.

37 Dederer (Anm. 16), S. 306. Vgl. auch Kirchhof, G., Die Kinderrechte des Grundgesetzes. Sollte die Verfassung zugunsten von Kindern geändert werden?, NJW 2018, S. 2690 (2691).

38 So etwa das Deutsche Institut für Menschenrechte (DIMR), Kinderrechte ins Grundgesetz. Kinder als Träger von Menschenrechten stärken, November 2016, S. 2, und Benassi/Eichholz (Anm. 4), S. 614 f.

39 Tobin, T., Article 4, in: ders. (Anm. 17), S. 108 (111).

40 Deutlich UN-Kinderrechtsausschuss, General Comment No. 13: The right of the child to freedom from all forms of violence, UN-Dok. CRC/C/GC/13, 2011, Rn. 65, 73; General Comment No. 19: Public budgeting for the realization of children's rights, UN-Dok. CRC/C/GC/19, 2016, Rn. $29 \mathrm{ff}$. 
lungsspielraum als auch das Ermessen der Vertragsstaaten nicht unempfindlich eingeschränkt. Eine Verpflichtung, Kinderrechte in der innerstaatlichen Rechtsordnung mit Verfassungsrang oder einem sonstigen normhierarchischen Vorranggebot auszustatten, ergibt sich daraus gleichwohl nicht. Eine effektive Umsetzung von Kinderrechten kann durchaus - und konventionskonform - bloß auf einfachgesetzlicher Ebene vorgenommen werden.

13. Vor diesem Hintergrund liegt der Vorwurf nahe, der UN-Kinderrechtsausschuss handele mit seinen unmissverständlichen Aufforderungen an die Vertragsstaaten zur verfassungsrechtlichen Implementierung von Kinderrechten über die ihm zugestandenen völkerrechtlichen Befugnisse hinaus, also ultra vires. ${ }^{41}$ Diesem Vorwurf kann aber schon deshalb entgegengetreten werden, weil die Auffassungen des Ausschusses rechtlich unverbindlich und letztlich auf einen Dialog mit dem Vertragsstaat ausgerichtet sind. ${ }^{42}$ Darüber hinaus ist zu bedenken, dass es dem UN-Kinderrechtsausschuss, der nicht notwendigerweise aus Juristen besteht, sondern interdisziplinär zusammengesetzt ist, ${ }^{43}$ weniger auf formaljuristische Normierungen denn viel stärker auf inhaltliche Perspektiven und Signalwirkungen ankommt. ${ }^{44}$ Maßgeblich ist es seiner Ansicht nach vor allem, dass das Kind in der innerstaatlichen Rechtsordnung als eigenständiges Rechtssubjekt begriffen und nicht lediglich als Schranke des elterlichen Rechts am Kind konzipiert wird. In welcher rechtsdogmatischen Form und an welchem normhierarchischen Standort dies geschieht, bleibt notwendigerweise den Vertragsstaaten überlassen. Ein Mandat zur rechtsverbindlichen Interpretation der UN-Kinderrechtskonvention hat der UN-Kinderrechtsausschuss nicht. ${ }^{45}$ Deshalb besteht keine völkerrechtliche Verpflichtung zur Änderung des Grundgesetzes. Hierüber sind sich sowohl die Bund-Länder-Arbeitsgruppe als auch der Referentenentwurf des BMJV im Ergebnis völlig zu Recht einig. ${ }^{46}$

\section{Bewertung des Referentenentwurfs des BMJV zur Einführung von Kinderrech- ten ins Grundgesetz aus völkerrechtlicher Perspektive}

14. Ob die Aufnahme einer Kinderrechtsklausel in das Grundgesetz im Allgemeinen und die im Referentenentwurf vorgeschlagene Formulierung eines neuen Art. 6 Abs. 1 a GG im Besonderen eine qualitative Verbesserung der Rechte von Kindern auf der innerstaatlichen Ebene bewirken würden, ist zweifelhaft. ${ }^{47}$ Denn nicht nur die Rechtsprechung des Bundesverfassungs-

41 Entsprechende Überlegung bei Dederer (Anm. 16), S. 306.

42 Vgl. Schmahl (Anm. 15), Art. 44/45 Rn. 8, 24.

43 Vgl. Art. 43 UN-KRK. Ferner siehe Dorsch, G., Die Konvention der Vereinten Nationen über die Rechte des Kindes, 1994, S. 275; Krappmann, L., Anspruch und Kerngehalte der Kinderrechtskonvention der Vereinten Nationen, in: Bielefeld, H. u.a. (Hrsg.), Jahrbuch Menschenrechte, 2010, S. 15 (16).

44 Vgl. mutatis mutandis zu den Erkenntnissen des UN-Behindertenrechtsauschusses Uerpmann-Wittzack, $R$., Die UN-Behindertenrechtskonvention in der Praxis des Ausschusses für die Rechte von Menschen mit Behinderungen, AVR 54 (2016), S. 181 (211).

45 Vgl. BVerfG, NJW 2018, S. 2619, Rn. 91, im Blick auf den UN-Behindertenrechtsausschuss.

46 Vgl. Abschlussbericht der Bund-Länder-Arbeitsgruppe (Anm. 2), S. 24, Rn. 2.1.2.; Referentenentwurf des BMJV (Anm. 3), S. 6.

47 Skeptisch auch Becker, F., Kinderrechte in die Verfassung? Zur Aufnahme eines Kindergrundrechts in das Grundgesetz, in: Uhle, A. (Hrsg.), Kinder im Recht. Kinderrechte im Spiegel der Kindesentwicklung, 2019, S. 251 (278 ff.); zurückhaltend wohl ebenfalls Radtke, H., Die Rechte von Kindern im Grundgesetz, DRiZ 2019, S. 56. 
gerichts und der Fachgerichte, ${ }^{48}$ sondern auch das einfache Recht orientiert sich seit der im Jahr 2010 erfolgten Rücknahme der deutschen Vorbehalte zur UN-Kinderrechtskonvention ${ }^{49}$ mittlerweile merklich an den subjektiven Rechten des Kindes. So sind in den letzten Jahren verschiedene kinderfreundliche Gesetze in Kraft getreten..$^{50}$ Dass dabei die Forderungen des Kindes oder seiner Interessenvertreter nicht immer uneingeschränkte Durchschlagskraft erzielt haben, liegt an der Konvention selbst, die als Menschenrechtsvertrag typischerweise auf Abwägungen von Rechten des Kindes mit Belangen seiner Eltern, sonstiger dritter Personen oder mit den Interessen der Allgemeinheit angewiesen ist. ${ }^{51}$

15. Es steht außer Frage, dass eine ausdrückliche Regelung von Kinderrechten im Grundgesetz den Rechtsanwendern die hohe und wertsetzende Bedeutung, die Kindern und ihren Rechten in der Rechtsordnung zukommt, nachdrücklich verdeutlichen würde. Deshalb mögen in der Tat psychologische und rechtssoziologische Erwägungen für die Aufnahme von Kinderrechten in das Grundgesetz sprechen. ${ }^{52}$ Die Fortentwicklung der bundesverfassungsgerichtlichen Judikatur zugunsten eines subjektiven Rechts des Kindes auf elterliche Pflege und Erziehung und zugunsten einer Stärkung des Selbstbestimmungsrechts und der Persönlichkeitsentfaltung des Kindes $^{53}$ lässt den rechtlichen Bedarf nach einer Grundgesetzänderung aber entfallen. Dies gilt umso mehr, als das Verhältnis zwischen Kinderrechten und Elternrechten in der Rechtsprechung des Bundesverfassungsgerichts feinsinnig austariert ist. ${ }^{54}$

16. Dementsprechend ist es konsequent, dass der Referentenentwurf des BMJV die Judikatur des Bundesverfassungsgerichts bei der sprachlichen Abfassung des neu in Art. 6 GG einzufügenden Abs. 1 a lediglich deklaratorisch aufgreift. Bemerkenswert ist jedoch, dass die gewählten Formulierungen hinter den textlichen Vorgaben der UN-Kinderrechtskonvention zurückbleiben. So soll das Wohl des Kindes nur ,angemessen“ berücksichtigt werden, während Art. 3 Abs. 1 UN-KRK ausdrücklich von einem Gesichtspunkt spricht, der „vorrangig“ zu berücksichtigen ist. Auch der Umstand, dass das Kind nach dem Referentenentwurf Anspruch auf rechtliches Gehör nur bei staatlichen Entscheidungen hat, die ,seine Rechte unmittelbar betreffen“, widerspricht dem Wortlaut von Art. 12 UN-KRK, wonach die Meinung des Kindes ,in allen das Kind berührenden Angelegenheiten“ angemessen zu berücksichtigen ist. Der Relativsatz des Referentenentwurfs des BMJV begrenzt das rechtliche Gehör des Kindes auf Verfahren, in denen eine Einzelfallentscheidung gefällt werden soll. Der Erlass generell-abstrakt wirkender Nomen soll das Beteiligungsrecht des Kindes nicht auslösen. ${ }^{55}$ Der UN-Kinder-

48 Vgl etwa BVerfGE 132, 134, Rn. 68; BVerfG, Beschluss vom 5.7.2013, 2 BvR 708/12, Rn. 22-24; BGH, FamRZ 2013, S. 1206 (1208); BVerwGE 145, 153 (155 f.); 146, 89 (96 f.); BVerwG, NVwZ 2013, S. 1493 (1495); LVerfG Bbg, NVwZ-RR 2011, S. 1514 (1514f.); OVG Berlin-Brandenburg, Urteil vom 29.11.2017, 1 B 19.15, Rn. 43; VG Berlin, FamRZ 2018, S. 399 (402). Zur Rechtsprechung der Fachgerichte überzogen kritisch Benassi (Anm. 4), DVBl. 2016, S. 618 f.

49 Vgl. BGBl. 2011 II S. 600.

50 Vgl. nur Schmahl (Anm. 28), S. $126 \mathrm{ff}$.

51 Ähnlich Dederer (Anm. 16), S. 322.

52 So etwa Hohmann-Dennhardt (Anm. 4), S. 186; Eichholz (Anm. 9), S. 20; Benassi/Eichholz (Anm. 4), S. 615. Auch der Referentenentwurf des BMJV nimmt auf diese Erwägung mehrfach Bezug, vgl. Referentenentwurf des BMJV (Anm. 3), S. 2, 5, 9.

53 Vgl. BVerfGE 24, 119 (124); 107, 104 (117); 121, 69 (92 f.); 132, 134, Rn. 68; 133, 50, Rn. 42 f.; vgl. auch Britz, G., Das Grundrechte des Kindes auf staatliche Gewährleistung elterlicher Pflege und Erziehung jüngere Rechtsprechung des Bundesverfassungsgerichts, JZ 2014, S. 1069.

54 So auch der Abschlussbericht der Bund-Länder-Arbeitsgruppe (Fn. 2), S. 25, Ziff. 2.1.2., sowie der Referentenentwurf des BMJV (Anm. 3), S. 8.

55 Vgl. Referentenentwurf des BMJV (Anm. 3), S. 13. 
rechtsauschuss geht jedoch im Blick sowohl auf Art. 3 UN-KRK als auch auf Art. 12 UN-KRK davon aus, dass sich das Mitspracherecht von Kindern auch auf Gesetzgebungsprozesse sowie auf Angelegenheiten beziehen soll, in denen die Interessen des Kindes nur mittelbar oder reflexhaft berührt sind. ${ }^{56}$

\section{Fazit}

17. Insgesamt geht das Vorhaben, Kinderrechte in das Grundgesetz aufzunehmen, einerseits insoweit über das völkerrechtliche Gebotene hinaus, als die UN-Kinderrechtskonvention einen Verfassungsrang von Kinderrechten nicht fordert. Andererseits bleibt die Ausgestaltung der vorgeschlagenen Grundgesetzänderung in Art. 6 Abs. 1 a GG hinter den völkerrechtlichen Vorgaben zurück, da die Norm das vom Bundesverfassungsgericht austarierte Verhältnis zwischen Eltern, Kindern und Staat nicht antastet. Aus verfassungspolitischem Blickwinkel ist dieser wirkungsneutrale Ansatz des Referentenentwurfs des BMJV durchaus zu begrüßen, da er der normhierarchischen Unterscheidung zwischen verfassungsrechtlichem Grundrechtsschutz und der UN-Kinderrechtskonvention als einfachem Bundesgesetz Rechnung trägt. Es verbleibt dann allerdings die Frage, weshalb eine derart wirkungsneutrale Grundgesetzänderung überhaupt angestrebt wird?

18. Der in Deutschland bislang vorherrschende Ansatz, kinderspezifische Regelungen im einfachen Gesetzesrecht dort festzuhalten, wo dies thematisch angezeigt ist, entspricht viel stärker der dogmatischen Tradition und führt gerade nicht per se zu einer Minderung der Rechte des Kindes. ${ }^{57}$ Soweit die deutsche Rechtsordnung weiterhin Defizite in der rechtlichen und sozialen Situation von Kindern enthält, weil diese zum Beispiel nicht über hinreichende (konsultative) Mitspracherechte in allen sie betreffenden Angelegenheiten ohne starre Altersgrenzen verfügen oder weil etwa die Ausstattung von Schulen, Jugendämtern und Kindertagesstätten partiell zu wünschen übrig lässt,$^{58}$ sollten zuvörderst das einfache Recht geändert und finanzielle Mittel bereitgestellt werden. Jedenfalls sollten Missstände nicht durch eine bloß „symbolische Grundgesetzänderung ${ }^{\text {“59 }}$ verschleiert werden.

Verf.: Prof. Dr. Stefanie Schmahl, Lehrstuhl für deutsches und ausländisches öffentliches Recht, Völkerrecht und Europarecht

Domerschulstraße 16

97070 Würzburg

E-Mail: schmahl@jura.uni-wuerzburg.de

$56 \mathrm{Zu}$ Art. 3 UN-KRK vgl. UN-Kinderrechtsausschuss, Concluding Observations: Turkey, UN-Dok. CRC/C/TUR/CO/2-3, 2012, Rn. 31; Australia, UN-Dok. CRC/C/AUS/CO/4, 2012, Rn. 32. Zu Art. 12 UN-KRK vgl. UN-Kinderrechtsausschuss, General Comment No. 12 (Anm. 8), Rn. 58.

57 Vgl. Schmahl, S., The Rights of the Child in Germany. The UN Convention on the Rights of the Child, and its Implementation in National Law, in: Schmidt-Kessel, M. (Hrsg.), German National Reports on the 19th International Congress of Comparative Law, 2014, S. 581 (586).

58 Näher Wapler, F., Gutachten zur Umsetzung und Anwendung der Kinderrechtskonvention in Deutschland, 2017, S. 4 f. Vgl. auch Janisch, W., Die Kinderrechte ins Grundgesetz zu schreiben, ist nicht genug, SZ (Online) vom 26.11.2019.

59 Begriff bei Wapler (Anm. 13), S. 513. 